\title{
Application On the Judgment of the Damage Position In the Bridge based Frequency Response Function
}

\author{
Xiang $\mathrm{Li}^{1, \mathrm{a}}$, Nianchun Deng ${ }^{2, b}$ \\ ${ }^{1}$ Department of Electrical Engineering, Guangxi Technological College of Machinery and Electricity, \\ Nanning, 530007, China \\ 2Department of Civil Engineering, Guangxi University, Nanning, 530004, China \\ axiangzi21th@hotmail.com, ${ }^{b}$ dengnch@163.com
}

Keywords: Structural Health Monitoring; Damage Index; Frequency Response Function

\begin{abstract}
A bridge structure may sustain damage either when subjected to severe loading like a strong earthquake or when its material deteriorates. Monitoring the structural health of a particular structural system involves a damage identification process that comprises detecting, locating, identifying type of, and estimating severity of, damage. The aim of this paper attempts to utilize frequency response function (FRF) as index in SHM for locating and estimating severity of damage to bridge structures. First, a numerical study, based on SAP2000 software, is employed to create simulated damage cases including single and multi-site(s) damage scenarios. Following, a novel FRF curvature method is developed and applied to locate damage. The simulation and experiment results expose that the proposed FRF index can locate damage correctly in single as well as multi-site(s) damage acceptably.
\end{abstract}

\section{Introduction}

Damage assessment of bridge structural health monitoring [1], can be divided into two parts, one for localized non-destructive inspection (Nondestructive testing), mainly refers to the case without destroying the subjects of structures, structural components of a single probe wherein the defect, the disadvantage is greater when the possibility of damage scope, manpower and time it takes to increase the relative and can not do on the structure integrity of the judge, to understand the impact on the overall structure damage; the other method as a whole of structural damage assessments, the use of pre-erected on the structure of the sensor to measure the dynamic response of a signal structure was affected by external disturbance (such as displacement, velocity, acceleration, etc.), then the signal using the obtained structure was subjected to damage recognition, comparison between different points in time and change parameters or signal response situations, this method can not only learn the location and extent of damage to the structure of, and further that the impact on the overall structure of the damage.

\section{Frequency Response Function}

The impulse response function [2] is the system of identifying the relationship of the resulting impulse response a function is a description of dynamic behavior of structural systems perturbed at the time domain and frequency response function with respect to the frequency domain is in the description of the frequency response function and impulse response function is converted to the frequency domain through a mathematical formula As a result, it is also a function of the frequency response with the dynamic characteristics of the structure of the system.

\section{Definition}

Suppose the unit pulse function make this system responds, then it is known as the unit impulse function; and if when an external force acting in response to any of the produce is called, relationship between external force and structural response is as follows [3]: 


$$
y(t)=\int_{-\infty}^{+\infty} h(\tau) x(t-\tau) d \tau
$$

Equations (1) is in the time domain and the structural response to any external force, according to the definition of swing points, both sides of Equations (1) take Fourier transform, then

$$
\int_{-\infty}^{+\infty} y(t) e^{-i \omega t} d t=\int_{-\infty}^{+\infty} \int_{-\infty}^{+\infty} h(\tau) x(t-\tau) e^{-i \omega t} d \tau d t
$$

The right hand side of Equations (2) is carried out after the Fourier transform, which is the natural frequency

$$
Y(\omega)=\int_{-\infty}^{+\infty} \int_{-\infty}^{+\infty} h(\tau) x(t-\tau) e^{-i \omega t} d \tau d t
$$

then, the equation (4) will be organized into

$$
Y(\omega)=\int_{-\infty}^{+\infty} h(\tau) e^{-i \omega \tau} d \tau \int_{-\infty}^{+\infty} x(u) e^{-i \omega u} d u
$$

Fourier transform for the two right-hand side, the last available following relation

$$
Y(\omega)=H(\omega) X(\omega)
$$

And that is the structure of the system frequency response function for this, by Equations (6) can be obtained relationship

$H(\omega)=Y(\omega) / X(\omega)$

Equations (7) is the basic definition of the frequency response function [4].

\section{Index}

Frequency response function of this paper consists of the following steps to establish indicators: structural acceleration response of the system by the Fourier transform into the frequency response function, the obtained varying degrees of damage to the integrity and the frequency response function under the map, then it can be treated as all structure Recognition extent under, then these results through substituting formula to form a frequency response function index value, and here the formula used to establish the index value is statistically common[5,6], respectively, Mean Absolute difference and Mean Square Difference, the formula are as follows:

$$
\begin{aligned}
& F_{i}^{A}=\left(\sum_{j=1}^{n}\left|D_{j}-I_{j}\right|\right) / n \\
& F_{i}^{S}=\left(\sum_{j=1}^{n}\left(D_{j}-I_{j}\right)^{2}\right) / n
\end{aligned}
$$

After the above Equations can be set up through the frequency response function of the index, while the index value to obtain a bridge structure of each point of division, the value of these indicators will depend staging point order, form a frequency response graph of a function indicators, The untreated indicator diagram called "the original frequency response function index map."

\section{Curvature}

Established using the equation (8) and (9) the original frequency response function indicator diagram can not clearly indicate the location of the damaged structures, then certainly in the judgment on the extent of damage will be affected, especially in multi-point destruction interaction under the influence, will allow the location and extent of the damage will be more difficult to judge, but because the index value is usually the point of damage will be greater than the index value on both sides of the point is not damaged, so that when viewed from the original index chart, the position of the point of failure are the figure most obvious point change in slope, so the use of this slope change point, improvements available curvature, curvature is the reference modal curvature method (Mode-shape Curvature Method) [7,8], this method was originally used for the floor-modal

$$
v_{i j}=\frac{\varphi_{i+1, j}-2 \times \varphi_{i, j}+\varphi_{i-1, j}}{h^{2}}
$$




\section{Establishment and analysis of numerical models}

In order to simplify the structure for analyze, where the use of steel frame bridge with six points as a sub-model analysis, force the process to make the steel frame that is only one direction of seismic force disturbance, and then measuring the direction of acceleration response signal, different structural damage loss of stiffness of each support section places do basis, ignoring the system mass and damping effect, as far as possible so that it is similar to a one-way analysis of shear frame, and the loss of rigidity of the support section is a way to shorten the column width simulation.

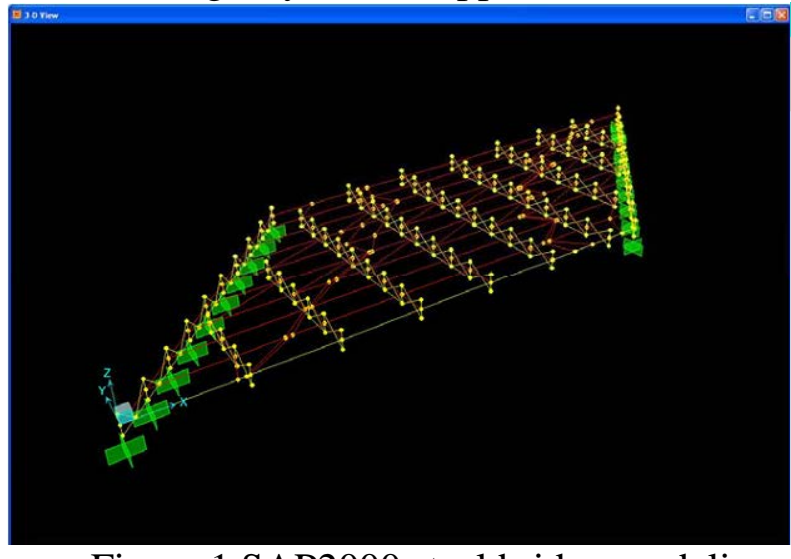

Figure 1 SAP2000 steel bridge modeling

\begin{tabular}{|c|l|r|r|r|r|r|r|}
\hline $\begin{array}{c}\text { TABLE: Modal Periods } \\
\text { OutputCase }\end{array}$ & StepType & StepNum & Period & Frequency & CircFreq & Eigenvalue \\
\hline Text & Text & Unitless & \multicolumn{1}{c|}{ Sec } & Cyc/sec & rad/sec & \multicolumn{1}{r|}{ rad2/sec2 } \\
\hline MODAL & Mode & 1 & 0.60903 & 1.642 & 10.317 & 106.43 \\
\hline MODAL & Mode & 2 & 0.202198 & 4.9457 & 31.074 & 965.62 \\
\hline MODAL & Mode & 3 & 0.12043 & 8.3036 & 52.173 & 2722 \\
\hline MODAL & Mode & 4 & 0.087414 & 11.44 & 71.879 & 5166.6 \\
\hline MODAL & Mode & 5 & 0.070851 & 14.114 & 88.682 & 7864.4 \\
\hline MODAL & Mode & 6 & 0.06274 & 15.939 & 100.15 & 10029 \\
\hline MODAL & Mode & 7 & 0.007087 & 141.1 & 886.55 & 785980 \\
\hline MODAL & Mode & 8 & 0.007084 & 141.15 & 886.89 & 786580 \\
\hline MODAL & Mode & 9 & 0.007079 & 141.27 & 887.64 & 787910 \\
\hline MODAL & Mode & 10 & 0.007069 & 141.47 & 888.89 & 790130 \\
\hline MODAL & Mode & 11 & 0.007056 & 141.73 & 890.51 & 793010 \\
\hline MODAL & Mode & 12 & 0.007044 & 141.96 & 891.98 & 795630 \\
\hline
\end{tabular}

Figure 2 Modal frequency data

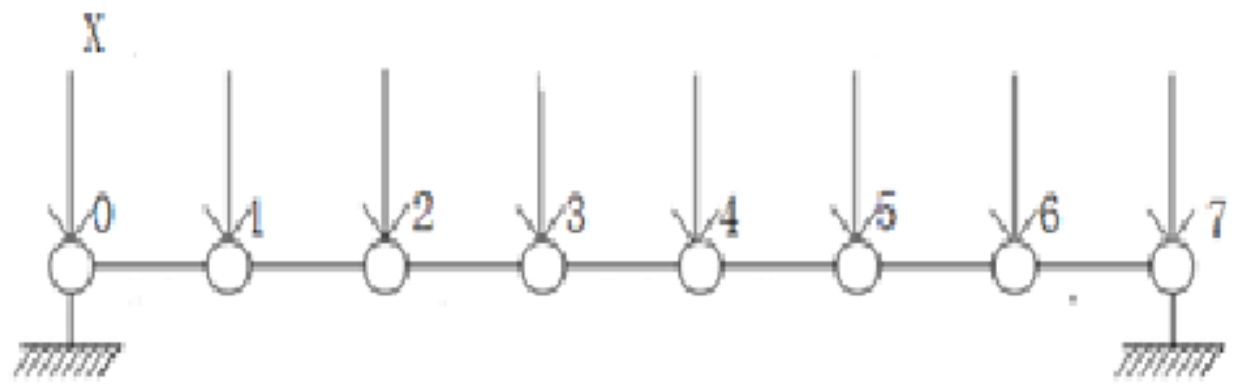

Figure 3 steel bridge model acceleration response collection location

Bridge structure is made of all-steel, the type of steel for the SAP2000 built of ASTM A992 steel, yield strength 3500kgf / cm2, frame appearance and frequency data shown in Figure 1 and Figure 2, which is divided into two beams member and post member section, section size beam member of $30 \mathrm{~mm} \times 240 \mathrm{~mm}$, beam length of $360 \mathrm{~mm}$; and section size column member is $15 \mathrm{~mm} \times 240 \mathrm{~mm}$, column height of $180 \mathrm{~mm}$ [9].

To identify the overall framework for damage, first of all it gives a certain degree of disturbance, followed by measuring the response of the perturbed framework. In the report by seismic forces as input acceleration response, seismic force data were Kobe 50gal, Kobe $100 \mathrm{gal}$ and El Central 50gal, El Central 100gal of four, which applies zero position number position in Figure 3, and then measuring acceleration response of each segment points, points were measured in Figure 3 No. 1 to No. 6 position, so get to the acceleration response at these numbers and different complete damage of the situation, it can be considered to be of preliminary damage identify this system.

\section{Improved curvature}

The above analysis, the use of the original frequency response function index maps, interaction on the location of the damage will lead to other locations to support the index value for each segment are large positive. then the curvature of the indicators in the form of a histogram showing diagra. Figure 4 is the first place of damage to $15 \%$ of the curvature index chart. In the case of the destruction of two, Figure 5 for the third place and fifth place at the same time 15 percent of the extent of damage to the curvature of the index chart, Figure 6 third place and fourth place also damaged 15 percent of the curvature of the index chart, so the curvature of the rear indicator diagram also has good results to help determine the location of damage. 


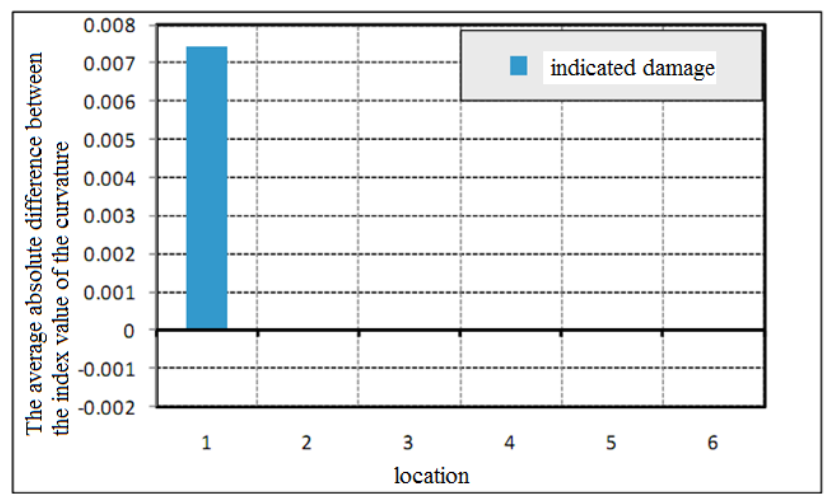

Figure 4 First place damaged $15 \%$ of the curvature indicators

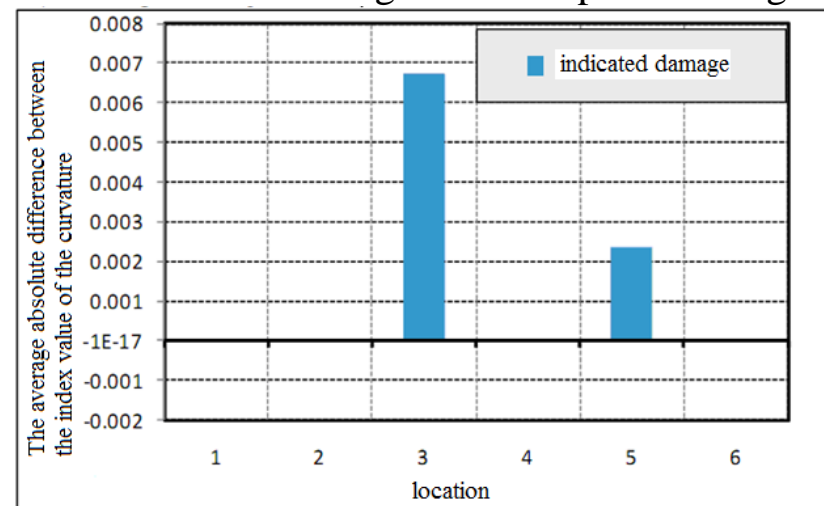

Figure 5 Third and fifth place damaged 15\%

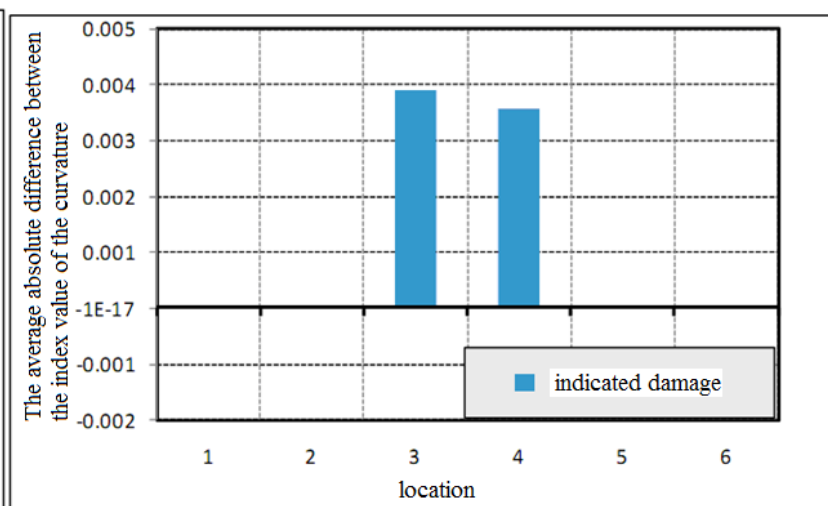

Figure 6 Third and fourth place damaged 15\%

\section{Summary}

According to the numerical analysis of the model, this general use of the frequency response of the original index is improved by curvature function established, and found either in the case of single-point or multi-point destruction, or damage in the same position of the case, which all will be the extent of damage Great, then the index value will be relatively increased property law is theoretically possible use of tools such intelligent identification.

\section{Acknowledgement}

This work was financially supported by the Guangxi Colleges and Universities Talents Scheme ( No. 40, Gui-Jiao-Ren [2011] ).

\section{References:}

[1] Z. D. Zhang, S. Chen. Journal of Marine Science and Application, 2007, 6(3): 1-5

[2] Z. Q. Qu. Mechanical Systems and Signal Processing, 2007, 21(1): 40-57

[3] Z. K. Peng, Z. Q. Lang, S. A. Billings. Journal of Sound and Vibration, 2007, 301(3-5): 777-788

[4] Z. K. Peng, Z. Q. Lang, S. A. Billings. Mechanical Systems and Signal Processing, 2008, 22(7): 1582-1594

[5] L. Pichler, H. J. Pradlwarter, G. I. Schueller. Computers \& Structures, 2008, 87(5-6): 332-341

[6] Q. B. Mao, Y. P. Nei, W. Zhang. Applied Mechanics and Materials. 2012, (160): 292-296

[7] Q. Mao, P. Stanislaw. Control of Noise and Structural Vibration. Germany: Springer, 2013: 255-280

[8] X. L. Guo, H. Y. Gao, S. H. Wang. Engineering Mechanics, 2012, 29(7): 56-83

[9] Y. P. Nie, Q. B. Mao, W. Zhang. Mechanical Analysis and Research, 2012, (2): 19-25 